\title{
LASIK for post penetrating keratoplasty astigmatism and myopia
}

\author{
Suzanne K Webber, Michael A Lawless, Gerard L Sutton, Christopher M Rogers
}

\begin{abstract}
Aims-To report the results of a series of patients who were treated with LASIK to correct post penetrating keratoplasty ametropia.

Methods-26 eyes of 24 patients underwent LASIK to correct astigmatism and myopia after corneal transplantation; 14 eyes also received arcuate cuts in the stromal bed at the time of surgery. The mean preoperative spherical equivalent was $-5.20 \mathrm{D}$ and the mean preoperative astigmatism was $8.67 \mathrm{D}$.

Results-The results of 25 eyes are reported. The mean 1 month values for spherical equivalent and astigmatism were $-0.24 \mathrm{D}$ and $2.48 \mathrm{D}$ respectively. 18 eyes have been followed up for 6 months or more. The final follow up results for these eyes are $-1.91 \mathrm{D}$ and $2.92 \mathrm{D}$ for spherical equivalent and astigmatism. The patients undergoing arcuate cuts were less myopic but had greater astigmatism than those not. The patients receiving arcuate cuts had a greater target induced astigmatism, surgically induced astigmatism, and astigmatism correction index than those eyes that did not. One eye suffered a surgical complication. No eyes lost more than one line of BSCVA and all eyes gained between 0 and 6 lines UCVA.

Conclusions-LASIK after penetrating keratoplasty is a relatively safe and effective procedure. It reduces both the spherical error and the cylindrical component of the ametropia. Correction of high astigmatism may be augmented by performing arcuate cuts in the stromal bed.

(Br f Ophthalmol 1999;83:1013-1018)
\end{abstract}

The presence of astigmatism and other ametropia is a major hurdle in the visual rehabilitation of patients who have undergone penetrating keratoplasty. The Australian Corneal Graft Registry ${ }^{1}$ suggests that three quarters of the patients undergoing corneal transplantation do so to improve visual acuity. It is therefore disappointing if postoperatively, the patient's acuity is limited by a high degree of ametropia. Thirty five per cent of patients in the registry in whom a value was recorded have a postoperative astigmatism of over $5 \mathrm{D}$. However, this value rises to $52 \%$ when keratometry values are considered. Eight per cent of grafts undergo some form of refractive surgery. Of these the majority are relaxing incisions with or without sutures or wound revision with a smaller number undergoing a laser procedure. We report a series of patients who underwent
LASIK after penetrating keratoplasty for the correction of high ametropia.

\section{Patients and methods}

PATIENTS

All patients undergoing LASIK for myopia or myopic astigmatism who had previously undergone penetrating keratoplasty in the operative eye were included. Only patients in whom spectacle or contact lens correction was unsatisfactory were considered for surgery. Twenty six eyes of 24 patients were included; 10 patients were female and 14 male. The average age of the patients at the time of LASIK was 37 years with a range of 19-73. Twenty three grafts had been performed for keratoconus, with one each further for childhood trauma, pseudophakic bullous keratopathy, and acanthamoeba keratitis. The mean interval between penetrating keratoplasty and LASIK was 8 years and 11 months, with a range of 1 year and 11 months to 23 years and 2 months. Four eyes had undergone previous ocular surgery since the corneal graft, one had a cataract extraction with intraocular lens, one had had a resuture of the graft host junction, and two had had incisional refractive keratectomies. All eyes had had the graft sutures removed a minimum of 10 months before LASIK.

Before surgery all eyes underwent a full ocular examination, including pachymetry.

\section{SURGICAL TECHNIQUE}

At the time of surgery amethocaine hydrochloride1\% (Chauvin Pharmaceuticals Ltd, Romford) was applied to the eye and the cornea marked with a pararadial marker with gentian violet. The suction ring was applied and the intraocular pressure was clinically assessed to be sufficiently elevated in all cases. The corneal cap was cut by an automated microkeratome (Automated Corneal Shaper, Chiron Technolas, Munich, Germany) with a depth limiting plate of $180 \mu \mathrm{m}$ fitted. All eyes were treated with a Summit Apex Plus excimer laser (Summit Pty Ltd, Waltham, MA, USA). In all cases the desired postoperative refraction was emmetropia. Throughout the study the refractive astigmatism was used for planning treatment and performing calculations. All cases received a toric laser correction using ablatable masks. In 14 eyes the astigmatic correction was augmented with arcuate cut incisions in the corneal bed after the laser had been performed. The arcuate cuts were performed with a guarded diamond knife set with a micrometer at $350 \mu \mathrm{m}$. Two arcs of $60^{\circ}$ to $80^{\circ}$ were performed in the axis of the steep meridian. 


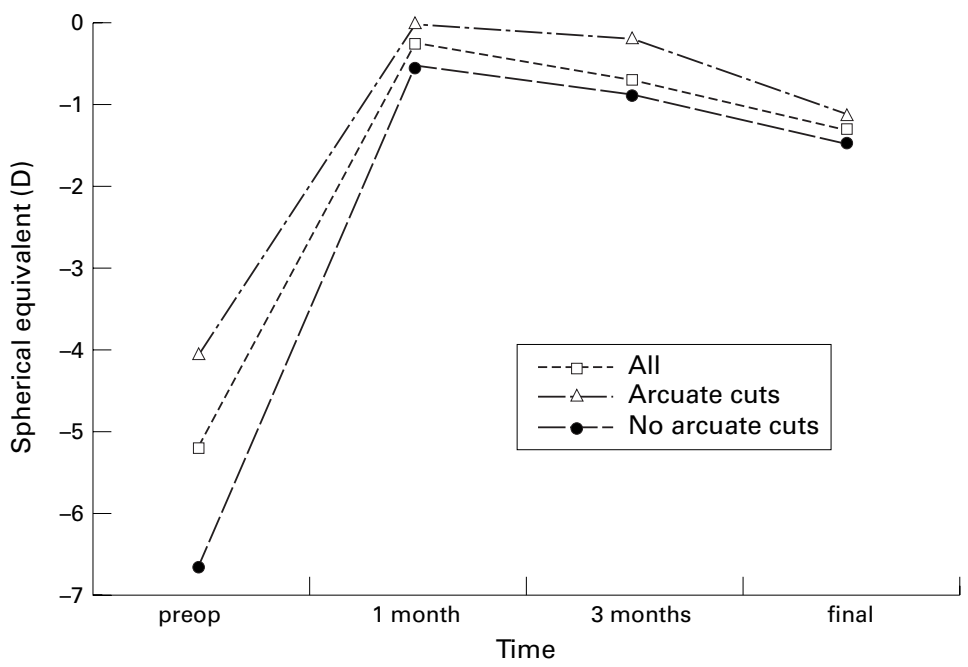

Figure 1 Change in spherical equivalent with time.

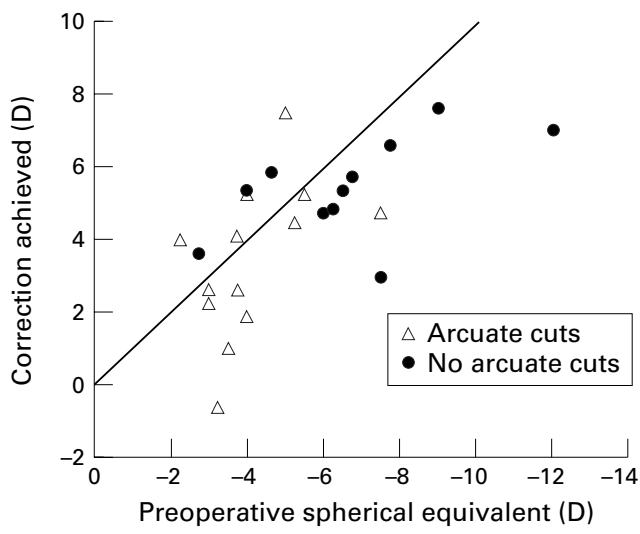

Figure 2 Preoperative spherical equivalent $v$ correction achieved at latest follow up.

The decision to perform arcuate cuts or not was based on an assessment of the cylindrical treatment possible with the laser (maximum of 5D) and constraints imposed by possible induced hypermetropia and optical zone size. If this was insufficient, arcuate cuts were performed.

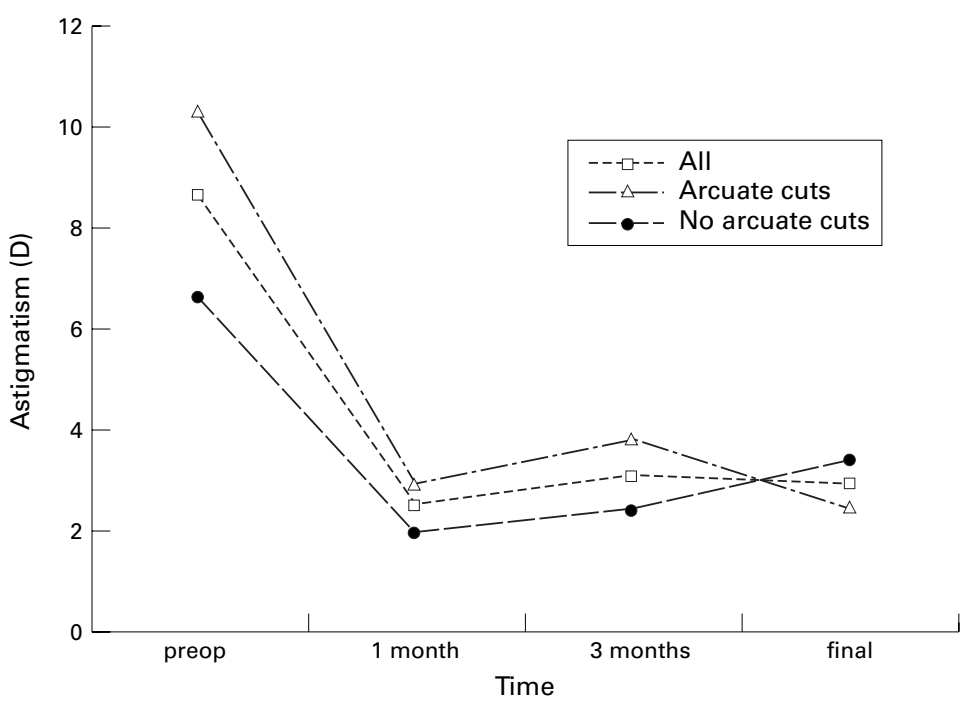

Figure 3 Change in astigmatism with time.
A complication was encountered with one patient where a perforation occurred at the time of arcuate cuts. This required resuturing and this patient has been excluded from the analysis. Therefore, the study population comprises 25 eyes of 23 patients. No other surgical complications were encountered. Specifically, there were no problems of graft dehiscence on application of the microkeratome suction ring. Postoperatively the patients wore an eye shield overnight. Tobramycin $0.3 \%$ (Alcon Laboratories (Australia) Pty Ltd, Sydney, Australia) and prednisolone acetate 1\% (Allergan Australia Pty Ltd, Sydney, Australia) three times daily were prescribed for the first week.

Postoperatively the patients were seen at 1 day, 1 week, 1, 3, 6, and 12 months. Not all patients attended all scheduled follow up appointments.

VECTOR ANALYSIS

The data were collected and analysed with the ASSORT computer software. This program has been designed to allow sophisticated analysis of surgically induced refractive change using vector analysis as described by Alpins. ${ }^{2}$ Briefly, the target induced astigmatism (TIA) is the amount of astigmatism that we hope to correct. It is measured at the corneal plane, and when the desired end result is emmetropia it is equal to the preoperative astigmatism. The surgically induced astigmatism (SIA) is the difference in the magnitude vectors between the preoperative and postoperative astigmatism. Thus, it represents the size of the change produced on the cornea by the treatment. The astigmatism correction index equals the SIA/TIA magnitude vectors. Thus if the ACI $=1$ the induced change equals the intended change. However, the induced change may have a reduced effect if it is not at the desired axis, therefore another index should also be considered. The difference vector (DV) is the further correction (magnitude and axis) required to attain the original goal from the result of the primary surgery; thus, a large DV is undesirable. Finally, the index of success (IS) may be considered, this represents DV/TIA and in this case a result as close to zero as possible is desirable.

STATISTICAL ANALYSIS

Analysis was performed on the groups as a whole and also split into two subgroups, those that received arcuate cuts and those that did not. The calculations were performed using sPSS software (SPSS Inc, Chicago, IL, USA) and the tests used were two tailed MannWhitney and Wilcoxon using a 5\% level for significance.

\section{Results}

PREOPERATIVE DATA

The mean spherical equivalent preoperatively was $-5.20 \mathrm{D}$ (range $-12 \mathrm{D}$ to $-2.25 \mathrm{D}$ (SD 2.31)). The mean preoperative astigmatism was $8.67 \mathrm{D}(2-15 \mathrm{D}$ (3.22)). The 14 eyes which received arcuate incisions at the time of surgery had a mean astigmatism of $10.29 \mathrm{D}$ and spherical equivalent of $-4.05 \mathrm{D}$ and in those 11 eyes 


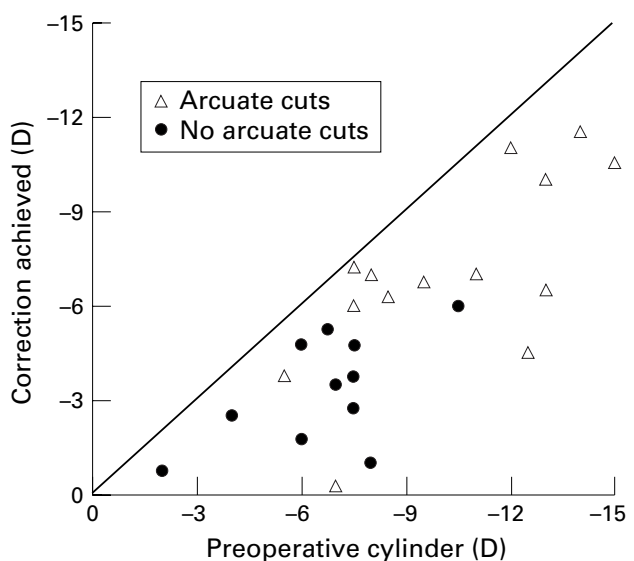

Figure 4 Preoperative astigmatism $v$ correction achieved at latest follow up.

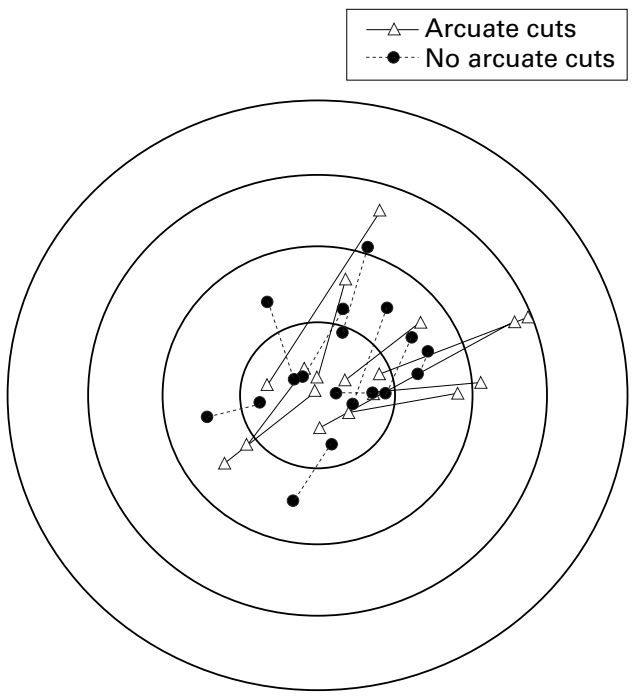

Figure 5 Doubled angle plot of preoperative and postoperative astigmatism. Each concentric circle represents $5 D$ astigmatism magnitude. The lines join the preoperative and postoperative values for a single eye. The ideal plot would have lines radiating from the centre like a cartwheel.

that did not have arc $\mathrm{T}$ cuts the mean astigmatism was $6.61 \mathrm{D}$ with a spherical equivalent of $-6.65 \mathrm{D}$. The two subgroups had significantly different preoperative values for both spherical equivalent and astigmatism when compared with each other $(p=0.003$ and $p=0.002)$. In 23 eyes the preoperative uncorrected visual acuity

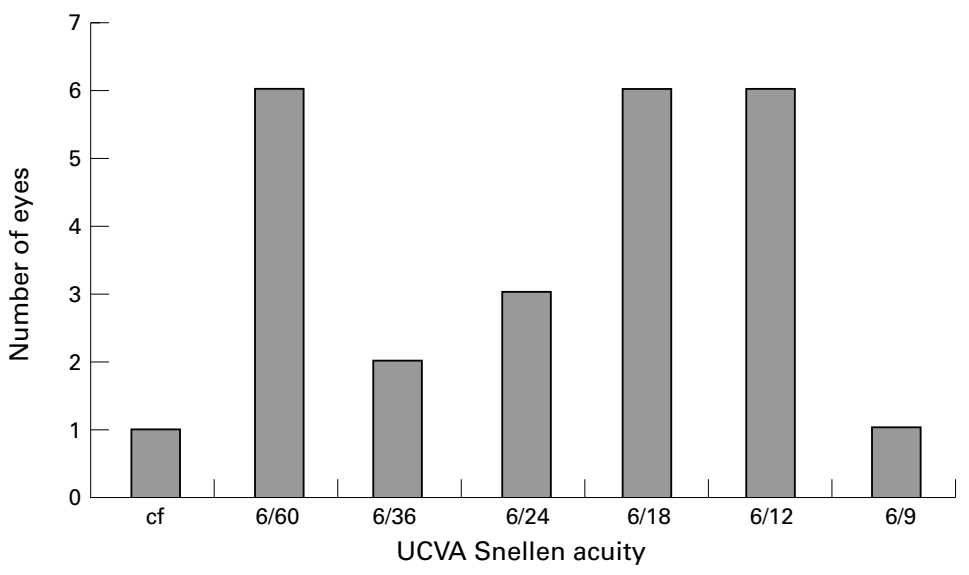

Figure 6 Postoperative UCVA at latest follow up.
(UCVA) was counting fingers and in the other two eyes it was 6/60. Preoperative best spectacle corrected visual acuity (BSCVA) ranged between $6 / 7.5$ and $6 / 24$.

\section{REFRACTIVE RESULTS}

At 1 month (22 eyes) the mean spherical equivalent was $-0.24 \mathrm{D}$ (range $-4.38 \mathrm{D}$ to $+2.88 \mathrm{D}$, SD 1.51). Six month follow up data are available for 11 eyes and 12 month data for seven eyes. No eye was seen for both visits; therefore, there are 18 eyes in which there is follow up data of 6 months or more. For statistical analysis these two groups have been combined and will be referred to as the final follow up data. The mean final spherical equivalent was $-1.31 \mathrm{D}(-5 \mathrm{D}$ to $+1.25 \mathrm{D}(1.63))$. There was no statistically significant difference between the two subgroups at final follow up, the means being $-1.47 \mathrm{D}$ and $-1.14 \mathrm{D}$ for the no arcuate cut and arcuate cut groups respectively. Reference to Figure 1 will demonstrate an apparent loss of effect from 1 month to final follow up. In actual fact when intra-eye change is analysed this was not statistically significant for the group as a whole or the arcuate cut subgroup, but there was a significant change for the no arcuate cut group $(p=0.047)$. Figure 2 illustrates the preoperative spherical equivalent plotted against the correction achieved. From this we can see that all patients, except one, had an improvement in their spherical equivalent.

The mean preoperative astigmatism was 8.67D, whereas at 4 weeks postoperatively this had reduced to $2.48 \mathrm{D}$ (range $0.25-8 \mathrm{D}$ (SD 1.90)) and at final follow up it measured 2.92D (0.25-7D (1.71)). The mean final astigmatism value for the arcuate cut subgroup was $2.44 \mathrm{D}$ and for the no arcuate cut group it was 3.39D. The difference between these two subgroups was not statistically significant. Figure 3 demonstrates the mean astigmatism over time. Like the spherical equivalent there appears to be loss of effect from 1 month to final follow up, but when intra-eye change is analysed there is no statistically significant change for the group as a whole or the arcuate cut subgroup. The change for the no arcuate cut group approached significance $(p=0.066)$. Figure 4 demonstrates the preoperative astigmatism against the correction achieved. From this we can see that no patient had an increase in the refractive astigmatism. Figure 5 is a doubled angle plot of the preoperative versus postoperative astigmatism. While the astigmatism was reduced in all cases, the fact that the lines are not radial shows that there is a shift in axis.

VISUAL ACUITY

At 1 month all the eyes had improved UCVA. Twenty eight per cent of eyes saw $6 / 12$ or better unaided at latest follow up (Fig 6). In no patients did the unaided visual acuity worsen. At latest follow up two eyes had not improved the UCVA, the remaining eyes had improved between 1 and 6 lines (Fig 7). Of those 18 patients who had reached at least 6 months' follow up, six had an UCVA of 6/12 or better.

Preoperatively $68 \%$ of eyes had a BSCVA of $6 / 12$ or better. At 1 month this had increased to 


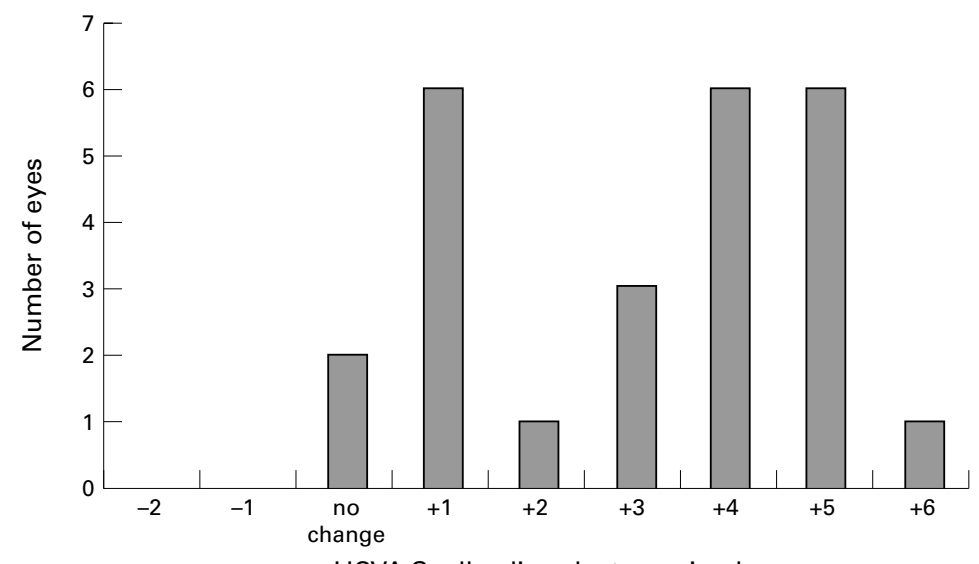

Figure 7 Snellen lines of UCVA lost or gained at latest follow up.

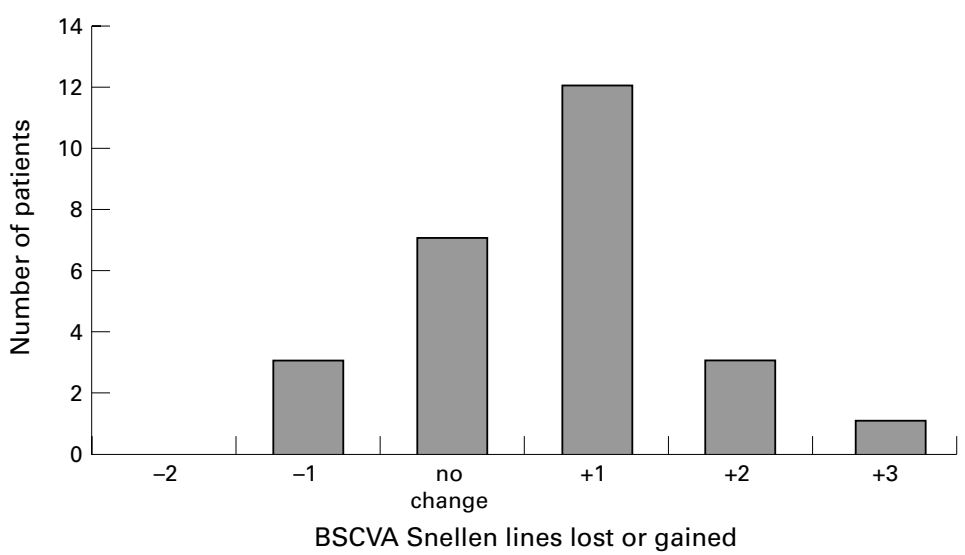

Figure 8 Snellen lines of BSCVA lost or gained at latest follow up.

$77 \%$ and at $6 / 12$ months $83 \%$. At latest follow up seven eyes had no change of Snellen BSCVA. Eleven patients had gained 1 line, three patients had gained 2 lines and one patient had gained 3 lines. Only three eyes lost 1 line of BSCVA and no eyes lost more than 1 line (Fig 8). Both subgroups showed a mean improvement in the BSCVA; however, the arcuate cut group showed a significant improvement from preoperatively $(p=0.03)$ unlike the no arcuate cut subgroup where the improvement was not statistically significant.

\section{VECTOR ANALYSIS}

The mean target induced astigmatism (TIA), with an aim of emmetropia for all patients, was $7.85 \mathrm{D}$ for the whole group (range 1.6213.1D), 9.36D for those receiving arcuate cuts, and 5.91D for those not (the difference being statistically different). At 1 month the mean surgically induced astigmatism (SIA) was $7.04 \mathrm{D}, 8.86 \mathrm{D}$, and $4.86 \mathrm{D}$ for the whole group, arcuate cuts, and no arcuate cuts respectively.
At final follow up, for those patients reaching 6 months or more, the SIAs were $6.10 \mathrm{D}, 8.31 \mathrm{D}$, and $3.88 \mathrm{D}$. These figures can be calculated into astigmatism correction index (SIA/TIA), or IS (DV/TIA). At final follow the ACI was $0.76,0.91$, and 0.60 for the whole group, arcuate cut, and no arcuate cut subgroups. The IS was $0.38,0.25$, and 0.50 for the whole group and two subgroups. The difference between the two subgroups for TIA, SIA, ACI, and IS are all statistically different at final follow up (Table 1). When the TIA for the no arcuate cut patients was assumed to equal the cylindrical treatment programmed into the laser the no arcuate cut group had final follow up values of 0.99 for the ACI and an IS of 0.54 .

\section{Discussion}

High residual refractive error can be a disappointing end point to an otherwise successful penetrating keratoplasty. ${ }^{3}$ If severe enough, it can prevent the patient attaining satisfactory visual acuity. Spectacles and contact lenses may be used to correct ametropia, but with high errors spectacles may cause unacceptable aberrations. In addition, contact lenses are difficult to fit after penetrating keratoplasty and are not without some risk to the graft. Thus, there is a cohort of patients that requires a further surgical procedure if they are to gain useful vision from their grafted eye.

Refractive procedures used in the past to reduce post-graft errors include retrephination, arcuate keratotomy, wedge resection, relaxing incisions, compression sutures, or a combination of these techniques. ${ }^{4-6}$ These generally, while having an effect on the astigmatic component of the ametropia, do little, if anything, to correct the spherical component. This, of course, is the concept of coupling whereby an incisional technique will flatten the steep meridian and steepen the flat meridian, but not change the overall corneal power. More recently the excimer laser has been used to perform photorefractive astigmatic keratectomy on corneal grafts. ${ }^{7-9}$ While this technique is able to correct myopia as well as astigmatism, the results have been somewhat unreliable and not without complication, including haze and scar formation. ${ }^{79}$

This series has demonstrated not only that LASIK after a corneal graft can be a safe and effective procedure, but also that augmentation with arcuate cuts at the time of surgery can lead to enhancement of the result. Our mean reduction in spherical equivalent from $-5.20 \mathrm{D}$ to $-1.31 \mathrm{D}$ is comparable with the largest previously reported series where the spherical equivalent in those reaching 6 months' follow up decreased from $-10.06 \mathrm{D}$ to $-4.13 \mathrm{D} .^{10}$

Table 1 Vector analysis

\begin{tabular}{|c|c|c|c|c|c|c|c|c|c|c|}
\hline & $\begin{array}{l}\text { Preop spherical } \\
\text { equivalent }\end{array}$ & $\begin{array}{l}1 \text { month } \\
\text { spherical } \\
\text { equivalent }\end{array}$ & $\begin{array}{l}\text { Final spherical } \\
\text { equivalent }\end{array}$ & $\begin{array}{l}\text { Preop } \\
\text { cylinder }\end{array}$ & $\begin{array}{l}1 \text { month } \\
\text { cylinder }\end{array}$ & Final cylinder & $T I A$ & Final SIA & Final $A C I$ & Final IS \\
\hline Whole group & -5.20 & -0.24 & -1.31 & 8.67 & 2.48 & 2.92 & 7.85 & 6.10 & 0.76 & 0.38 \\
\hline Arcuate cuts & $-4.05^{\star}$ & 0.00 & -1.14 & $10.29^{\star}$ & 2.92 & 2.44 & 9.36 * & $8.31^{\star}$ & $0.91^{\star}$ & $0.25^{\star}$ \\
\hline No arcuate cuts & $-6.65^{\star}$ & -0.53 & -1.47 & $6.61^{\star}$ & 1.95 & 3.39 & $5.91 \star$ & $3.88^{\star}$ & $0.60^{\star}$ & $0.50^{\star}$ \\
\hline
\end{tabular}

^Statistically significant difference between two subgroups.

All values are in dioptres (D) 
Other reports of LASIK after penetrating keratoplasty (PKP) also demonstrate a general reduction in spherical equivalent ${ }^{11}{ }^{12}$; however, the change in spherical equivalent was not as great as that generally found with normal corneas. $^{13}$

The reduction in astigmatism from a mean of $8.67 \mathrm{D}$ to $2.96 \mathrm{D}$ is very satisfactory. It is gratifying to note that while the arcuate cut subgroup started with a higher preoperative astigmatism (and therefore TIA), they also achieved a higher SIA. They also attained a higher ACI (SIA/TIA) than the no arcuate cut subgroup, thus demonstrating that the incisions contributed to the correction of the astigmatism. An ACI of 0.91 means that for the arcuate cut subgroup, the achieved astigmatic effect was only slightly smaller than that intended. The ACI for the no arcuate cut subgroup was lower at 0.60 . However, this might be predicted because the laser used has a maximal correction of $5 \mathrm{D}$ allowing the full astigmatism to be corrected in only some cases, thus rendering it unlikely that the SIA would approach the TIA. To compensate for this, when the TIA for the no arcuate cut subgroup was adjusted to be equal to the programmed laser correction (as opposed to emmetropia) the ACI was calculated to be 0.99 . This would suggest that the laser is highly effective at performing astigmatic ablations, but the clinical result is reduced by axis discrepancies.

Few papers have previously reported on the use of LASIK in the treatment of postkeratoplasty astigmatism. One report on the use of LASIK to correct postoperative myopia and astigmatism ${ }^{11}$ details a small series of patients and shows a good correction of the myopic component, but a disappointing response to fairly modest astigmatic components (mean cylinder changed from $-2.87 \mathrm{D}$ to $-3.50 \mathrm{D}$ postoperatively). The same authors later updated their list of post-PKP patients undergoing LASIK. ${ }^{10}$ In this revised list of 15 patients only eight had reached the 6 month stage. Of these eight patients the spherical component was generally effectively treated. However, the correction of the astigmatism was more variable with half of the patients at 6 months having no change or an increase in the refractive cylinder.

It should be noted that in this study the BSCVA reported was that level of vision achieved in the office with the refractive correction pushed to the limit. It does not necessarily represent the patient's vision with the spectacle correction that could be worn and tolerated. This is the reason why we performed surgery on some eyes with apparently good preoperative visual acuity. For example, one patient with a preoperative BSCVA of $6 / 9$ only achieved this with a correction of $+1 /-12$. Obviously, her acuity with a wearable correction would have been considerably less than $6 / 9$.

It is reassuring that no patients lost more than one line of BSCVA. Corneal scarring leading to the loss of 3 or 4 lines is a serious complication associated with photoastigmatic keratectomy. ${ }^{79}$ Our patients demonstrated a modest improvement in BSCVA. There are various possible reasons for this. Firstly, in those patients in whom a significant degree of myopia was corrected a magnification effect may be observed. Secondly, by creating arcuate cuts in the stromal bed irregular forces acting on the central cornea may be relieved thus reducing the amount of irregular astigmatism. The finding that the arcuate cut subgroup improved more than the no arcuate cut subgroup would be consistent with this suggestion. Indeed, some surgeons perform a keratectomy alone in the first instance and then perform laser ablation as a second procedure only if required. Alternatively, the improvement in visual acuity could simply be because of the reduced spectacle lens power and so less potential for aberrations exists. It is reported in the Australian Corneal Graft Registry that $78 \%$ of grafts performed for keratoconus see $6 / 12$ or better with their usual correction. ${ }^{1}$ While the follow up interval and exact correction is unspecified, it is likely that our patients are seeing at least as well postoperatively as an unselected sample.

However, we feel that although it is reassuring that patients do not, on the whole, lose BSCVA, it should be our surgical goal for the patient to achieve good uncorrected acuity. Just as the goal of cataract surgery has evolved from simply achieving anatomical integrity, through the need for good corrected acuity, to a desire for normal uncorrected vision, so too should our goal move. Previous studies have failed to report uncorrected visual acuity results, possibly because they are generally not very good. The finding that one third of our patients at 6 months or later see $6 / 12$ or better unaided is pleasing but we should not become complacent. The equivalent figure for normal corneas undergoing toric myopic LASIK is almost $80 \% .{ }^{13}$ Two thirds of the study eyes achieved functional unaided acuity measuring $6 / 24$ or better. We would like to suggest that unaided acuity should be included as an outcome measure in future reports of the refractive results of corneal transplantation.

We found this procedure to be relatively free of complications. One patient sustained a perforation at the time of arcuate cut incisions. This would not always cause significant complications, but in this patient wound resuture was required. There is also the risk of penetrating keratoplasty wound rupture on application of the microkeratome suction ring when an intraocular pressure of approximately 60 $\mathrm{mm} \mathrm{Hg}$ is created. All eyes sustained this increased pressure without apparent adverse effect. Complications noted by other authors include bleeding into the interface after the keratome passed, locking of the keratome due to an old nylon corneal suture, ${ }^{11}$ and worsening of the refractive error. ${ }^{10}$ One theoretical risk is that of inciting a rejection episode. This has not occurred so far, postoperative steroids probably contribute to ensuring against this.

The timing of further refractive surgery may be debated. Our patients had their penetrating keratoplasty at least 23 months before LASIK. Other authors recommend waiting $2-3$ years. ${ }^{12}$ 
However Lam et al feel that some younger patient require more urgent correction of the anisometropia ${ }^{14}$ and they performed LASIK at 17 and 23 months without incident. They suggest that clinical examination of wound healing signs and relative youth of the patient may allow it to be done safely earlier than that previously recommended.

In conclusion, we suggest that LASIK after penetrating keratoplasty is a safe and very effective procedure. It offers a valuable option in the management of post keratoplasty ametropia. Furthermore, higher degrees of astigmatism may be corrected by performing arcuate cuts in the stromal bed of the LASIK flap.

The authors would like to acknowledge the contribution to this paper of Dr S Montano who wrote the program allowing automatic production of the doubled angle plot (Fig 5).

1 Williams KA, Muehlberg SM, Lewis RF, et al, eds. The Australian Corneal Graft Registry 1996 Report. Adelaide: Flinders Medical Centre.

2 Alpins NA. A new method of analysing vectors for changes in astigmatism. F Cataract Refract Surg 1993;19:524-33.

3 Troutman RC, Lawless MA. Penetrating keratoplasty for keratoconus. Cornea 1987;6:298-305.
4 Kruger RR, Landry RJ, Assil KK, et al. Retrephination keratoplasty for high astigmatism after penetrating keratoplasty. F Refract Surg 1996;12:806-8.

5 Whitehouse G, Sutton G, Lawless MA, et al. Refractive keratoplasty for post-graft astigmatism. Aust NZ $\mathcal{F}$ Ophthalmol 1994;22:243-7.

6 Kirkness CM, Ficker LA, Steele ADM, et al. Refractive surgery for graft-induced astigmatism after penetrating keratoplasty for keratoconus. Ophthalmology 1991;98: 1787-92.

7 Tuunanen TT, Ruusuvaara PJ, Uusitalo RJ, et al. Photoastigmatic keratectomy for correction of astigmatism in corneal grafts. Cornea 1997;16:48-53.

8 Nordan LT, Binder PS, Kassar BS, et al. Photorefractive keratectomy to treat myopia and astigmatism after radial keratotomy and penetrating keratoplasty. 7 Cataract Refract Surg 1995;21:268-73.

9 Lazzaro DR, Haight DH, Belmont SC, et al. Excimer laser keratectomy for astigmatism occurring after penetrating keratoplasty. Ophthalmology 1996;103:458-64.

10 Arenas E, Garcia J. LASIK for myopia and astigmatism after penetrating keratoplasty. Response. F Refract Surg 1997;13: $501-2$

11 Arenas E, Maglione A. Laser in situ keratomileusis for astigmatism and myopia after penetrating keratoplasty. F Refract Surg 1997;13:27-32.

12 Parisi A, Salchow DJ, Zirm ME, et al. Laser in situ keratomileusis after automated keratoplasty and penetrat-

13 Fraenkel GE, Webber SK, Sutton GL, et al. Toric LASIK for myopic astigmatism. $\mathcal{F}$ Refract Surg (in press).

14 Lam DSC, Leung ATS, Wu JT, et al. How long should one wait to perform LASIK after PKP? F Cataract Refract Surg 1998;24:6-7. 